\title{
A Data Mining approach for Prediction Modeling
}

\author{
Rzgar Sirwan Raza \\ Department of Computer Science, College of Science and Technology \\ University of Human Development \\ Qaradagh, Sulaymaniyah, Kurdistan Region, Iraq
}

\begin{abstract}
In the present work, a data mining approach is highlighted, a prediction optimization data mining approach CuS (Cuckoo search) is chosen for performing prediction modeling, a data mining prediction analysis model is formulated based on $\mathrm{CuS}$ is presented in this work. The result of the model formulated is then compared with the result produced on the similar set of input on the traditional optimization problems. While comparing the results it was observed that the result produced by the presented model is much closer to the reality.
\end{abstract}

Keywords: DM: Data Mining; CuS:Cuckoo search; DW- Data warehouse.

\section{I.INTRODUCTION}

Data Mining is a logical procedure considered to explore data to investigate the reliable patterns and/or efficient associations between dependent and independent variables, and then to confirm the conclusion by applying to detected patterns on the available data in the Data warehouse data. The vital goal of data mining is to predict - and predictive mining is the most regular type of data mining and also, prediction modeling is very use full in all type of business applications [5,6]._Cuckoo search (CS) is a prediction optimization data mining approach developed in the year 2009[1]. It was stimulated by the brood parasitism. Few host birds can connect direct disagreement with the interfering cuckoos. Such as, if a host bird were found that the eggs are not of the host bird, the host bird will throw these eggs away. Few of the cuckoo species have evolved in such a way that non-male parasitic cuckoos are frequently particular in the impersonation in colors and blueprint of the eggs of a few selected host species [2].

In this work, the next section depicts the hypothesis of the proposed model; section-3 shows the comparison of current model results with the one of traditional model results on the similar set of data; after this section next section shows the conclusion and future scope of this work and the last section of paper is references section.

\section{HYPOTHESIS}

The proposed model is based on the Cuckoo search, it is used for only for estimation of the $\%$ of public transport in South Delhi, India region.

The input for the models is south Delhi Population(P), per-captia income of the south Delhi region(PC), and the output is the trend produced in term of $\%$ of public transport chosen by the population (PPT)[3].

$\mathrm{PPT}=$ function $(\mathrm{P}, \mathrm{PC})-(1)$

\section{RESULT COMPARISON}


Table 3.1 shows the data, while implementation of the model the following table data is used to take the input of the proposed model shown in equation 1.

\begin{tabular}{|c|c|c|c|}
\hline Year & Population & $\begin{array}{c}\text { Per capita income of } \\
\text { Area }\end{array}$ & Percentage of Mode of public transport \\
\hline 2001 & 2267023 & 40000 & 69.8 \\
\hline 2011 & 2733752 & 120352 & 65.9 \\
\hline 2015 & 3280502 & 250000 & 59.1 \\
\hline
\end{tabular}

Table 3.1 South Delhi trends data [4]

Similar exercise was done with the linear programming based approach; the result of the comparison is shown in the figure 3.1 below:

\section{5}

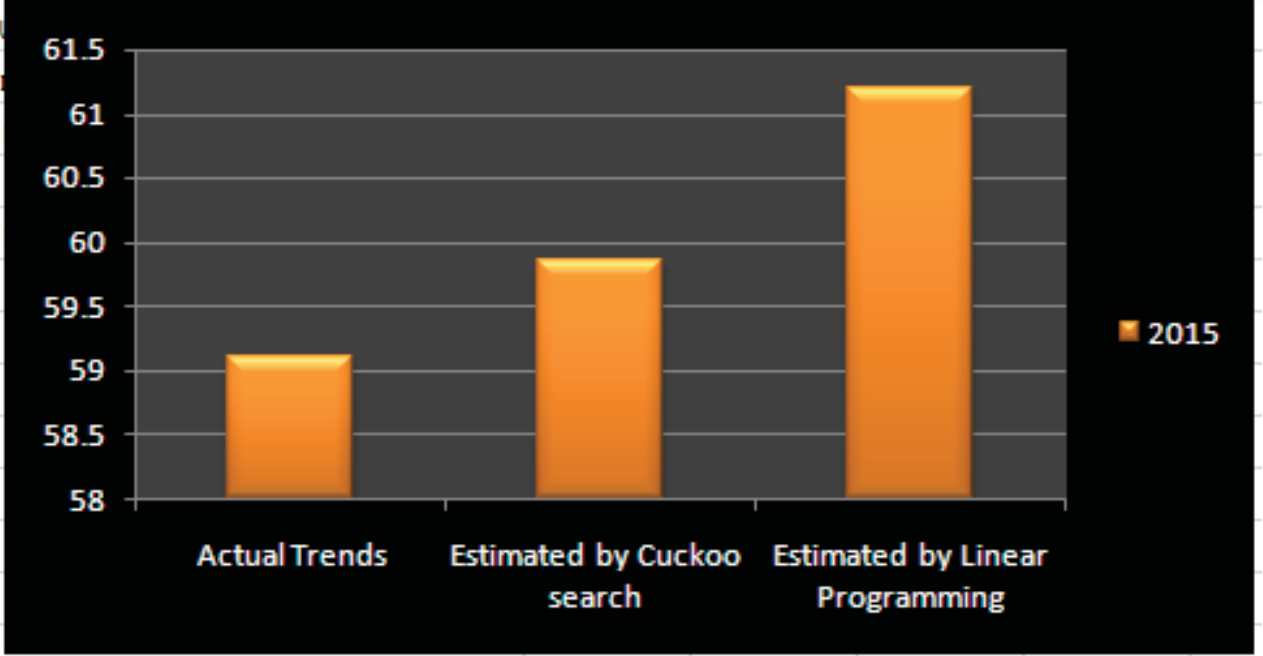

Figure 3.1 Result comparison

While looking at the Figure 3.1 one can easily be observed that the result produced by the proposed model are very close to the Actual trends on the other hand result produced by the traditional approach are not as closer to the Actual trends.

\section{CONCLUSION AND FUTURE SCOPE}

The result of the model formulated is compared with the result produced on the similar set of input on the traditional optimization problems. While comparing the results it was observed that the result produced by the presented model is much closer to the reality. This model can be enhanced for the geographically bigger areas.

\section{REFERENCES}

[1] X.-S. Yang; S. Deb (December 2009). Cuckoo search via Lévy flights. World Congress on Nature \& Biologically Inspired Computing (NaBIC 2009). IEEE Publications. pp. 210-214.

[2] R. B. Payne, M. D. Sorenson, and K. Klitz, The Cuckoos, Oxford University Press,[2005]

[3] Goel Shivendra, Singh, Sinha, "Trip Distribution Model for Delhi Urban Area Using Genetic Algorithm” IJCES Volume 2 Issue 32012

[4] Internet Sources, www.google.com

[5] http://documents.software.dell.com/statistics/textbook/data-mining-techniques\#pdm 
[6] Khan, Imran, et al. "Data model for Big Data in cloud environment." Computing for Sustainable Global Development (INDIACom), 2015 2nd International Conference on. IEEE, 2015. 